\title{
Observation of femtosecond carrier thermalization time in indium nitride
}

\author{
Shih-Ze Sun, ${ }^{1}$ Yu-Chieh Wen, ${ }^{1}$ Shi-Hao Guol, ${ }^{1}$ Hong-Mao Lee, ${ }^{2}$ Shangjr Gwo, ${ }^{2}$ and \\ Chi-Kuang Sun ${ }^{1,3, a)}$ \\ ${ }^{1}$ Department of Electrical Engineering and Graduate Institute of Photonics and Optoelectronics, National \\ Taiwan University, Taipei, Taiwan 10617, Republic of China \\ ${ }^{2}$ Department of Physics, National Tsing-Hua University, Hsinchu, Taiwan 30013, Republic of China \\ ${ }^{3}$ Research Center for Applied Sciences, Academia Sinica, Taipei, Taiwan 115, Republic of China
}

(Received 9 December 2007; accepted 13 April 2008; published online 18 June 2008)

\begin{abstract}
Ultrafast carrier thermalization in $n$-type indium nitride $(\mathrm{InN})$ with an electron concentration of $3.8 \times 10^{18} \mathrm{~cm}^{-3}$ was investigated by femtosecond transient transmission measurements at room temperature with different wavelengths. An extremely fast carrier external thermalization time on the order of $400 \mathrm{fs}$ was observed, which is much faster than all previous reports. This observed femtosecond thermalization time is consistent with a prediction based on a Coulomb screening effect. Through wavelength dependent and power dependent studies, even with a 400 fs thermalization time, we did not observe any evidence of the existence of the hot phonon effect, which agrees with a recent report that a longitudinal optical phonon lifetime could be shorter than $300 \mathrm{fs}$ in specific InN samples. (C) 2008 American Institute of Physics. [DOI: 10.1063/1.2940737]
\end{abstract}

\section{INTRODUCTION}

Indium nitride (InN), a wurtzite group-III-nitride semiconductor, has attracted much attention recently because the availability of high crystalline quality promotes the discovery of the fundamental band gap energy to be around $0.7-0.8$ $\mathrm{eV}^{1}$ With a narrow direct band gap in $\mathrm{InN}$, the emitting spectral range of the ternary alloys $\mathrm{Ga}_{x} \mathrm{In}_{1-x} \mathrm{~N}$ can extend from ultraviolet to infrared, making the InN-based material highly potential to be applied in optoelectronics, especially in solar cells. InN is also known to have some excellent properties for high-speed electronic device development, such as high electron mobility and high saturated drift velocity. Although many physical, chemical, and thermal characterizations in InN have already been extensively investigated up to date, the ultrafast carrier relaxation mechanism is still not well understood, and this knowledge is crucial in device applications.

Being highly polarized with a large longitudinal optical (LO) phonon energy of $73 \mathrm{meV}^{2}, \mathrm{InN}$ is expected to have a strong coupling between carriers and polar optical phonons, of which the interaction should dominate the hot carrier relaxation. Experimentally, Chen et al., ${ }^{3}$ Zanato et al., ${ }^{2}$ and Pačebutas et al. ${ }^{4}$ have reported observations of carrier cooling time, electron-LO phonon scattering time, and optical bleaching recovery time, which were all rather long and far from the theoretical electron-LO phonon scattering time predicated to be sub-100 $\mathrm{fs}^{2}$. The authors attributed the results to the hot phonon effect. Recently, the study by Wen $e t$ $a l .,{ }^{5}$ concerning the carrier density dependence of the hot carrier cooling time at high carrier concentrations, found that the serious reduction of the thermalization rate is mainly caused by the electron-plasma screening effect rather than the hot phonon effect. Nevertheless, the controversy of the hot carrier relaxation behavior in InN has not ceased because

\footnotetext{
${ }^{a)}$ Electronic mail: sun@cc.ee.ntu.edu.tw. Tel.: +886-2-33665085. FAX: +886-2-33663614.
}

two recent reports concluded against the study by Wen et $a .^{5}$ One is that Tsai et al..$^{6}$ observed the carrier relaxation time revealed the density dependence $n^{-0.5}$, so that they attributed the result to electron-electron scattering. The other is that Jang et al. ${ }^{7}$ attributed their result to the hot phonon effect by time-resolved photoluminescence (TRPL) study. In fact, for the former that performed transient reflectivity measurements, the measurements were unable to probe the cooling behaviors of the thermalized carriers due to the extremely high probe photon energy. As for the latter that extracted the temperature evolution of hot carriers from TRPL measurements, it was incapable of retrieving such fast cooling times due to lacking sufficient time resolution. Even though the reported experimental result is reliable, their misunderstanding of the screening effect resulted in the reasoning error in conclusion, and on the contrary, their result actually agreed with the prediction of the screening effect, rather than the hot phonon effect. In spite of these two untenable reports, in an InN material system, nonequilibrium phonons are still expected to play an important role in hot carrier relaxation if the Coulomb screening effect becomes minor.

In this study, a high quality InN sample, with a doping density low enough to reveal a dramatic difference in screening-dominated carrier cooling time, was used to investigate the effects between the screenings and the hot phonons. By means of more correct experimental methods, namely, the time-resolved near-band-gap transmission spectroscopy under low excitation, we observed an extremely fast carrier external thermalization time on the order of $400 \mathrm{fs}$, which is much faster than all previous experimental reports. This observed femtosecond thermalization time is consistent with the prediction based on a Coulomb screening effect. Through wavelength dependent and power dependent studies, even with a 400 fs thermalization time, we did not observe any evidence of the existence of the hot phonon effect, which agrees with recent reports that a LO phonon lifetime could be shorter than $300 \mathrm{fs}$ in specific InN samples. ${ }^{8,9}$ 


\section{SAMPLE PREPARATION}

The wurtzite InN sample investigated in this work was prepared by plasma-assisted molecular beam epitaxy. Using the epitaxial $\beta-\mathrm{Si}_{3} \mathrm{~N}_{4} / \mathrm{AlN}$ double-buffer layer technique, an AlN layer with a thickness of $120 \mathrm{~nm}$ and an InN layer with a thickness of $840 \mathrm{~nm}$ were grown on a $\mathrm{Si}(111)$ substrate. ${ }^{10}$ An unintentional $n$-type doping concentration of 3.8 $\times 10^{18} \mathrm{~cm}^{-3}$ was determined by the Hall measurement at room temperature (RT).

\section{EXPERIMENT}

To investigate ultrafast carrier dynamics, femtosecond time-resolved experiments were performed at RT using a degenerate pump-probe technique with an optical parametric oscillator pumped by a Ti:sapphire laser. The central wavelength of the laser pulses to excite and probe the carrier populations was tuned between 1300 and $1430 \mathrm{~nm}(0.953$ $0.866 \mathrm{eV}$ ), which were slightly higher than the band gap energy of InN, with a pulsewidth of around $180 \mathrm{fs}$. Using two lenses with different focal lengths to focus beams onto the sample, the excitation intensity can be controlled in a wide range. The focused spots of the pump and probe beams were kept the same with 40 and $8 \mu \mathrm{m}$ radii for two lenses, measured by a pinhole. The pump fluence was varied from 0.17 to $124 \mu \mathrm{J} / \mathrm{cm}^{2}$ to observe carrier dynamics with a photoexcited carrier density from $1.4 \times 10^{16}$ to $4.2 \times 10^{18} \mathrm{~cm}^{-3}$, corresponding to the increase in density than the background electron density of $3.8 \times 10^{18} \mathrm{~cm}^{-3}$. When the photoexcited carrier densities were lower than $2 \times 10^{16} \mathrm{~cm}^{-3}$, the observed carrier kinetics were unchanged and were treated as being in the perturbative regime. An optical chopper was used to modulate the pump beam at $800 \mathrm{~Hz}$ to improve the signal-to-noise ratio. The reflection and transmission power were also measured for the purpose of estimating the photoexcited carrier density. Since the absorption experiment directly measures the quantity related to the sum of the distribution function of electrons and holes, ${ }^{11}$ the absorption changes relevant to the component of the carrier thermalization can be calculated to be dominated by electrons under our experimental conditions.

\section{EXPERIMENTAL RESULTS AND DISCUSSION}

In order to study the hot carrier cooling process, we first performed transient transmission experiments by choosing excitation photon energies near and above the absorption edge under low excitation to slightly perturb the background carrier system in InN. Figure 1 shows the measured transient transmission changes of excitation photon energies from 0.866 to $0.953 \mathrm{eV}$ with a fixed photoexcited carrier density of $1.4 \times 10^{16} \mathrm{~cm}^{-3}$. Through a convolution fitting procedure, all traces can be represented by an impulse response function of the form $u(t)\left[a_{1} \exp \left(-t / \tau_{1}\right)+a_{2} \exp \left(-t / \tau_{2}\right)\right]$, where $u(t)$ is the unit step function. The faster exponential decay component with a time constant $\tau_{1}$, on the order of $400( \pm 100)$ fs, is found independent of the excitation photon energies and is much faster than the slower time constant $\tau_{2}$, which is on the order of or longer than 100 ps. By changing the pumped carrier density, we can confirm that all traces in Fig. 1 are in

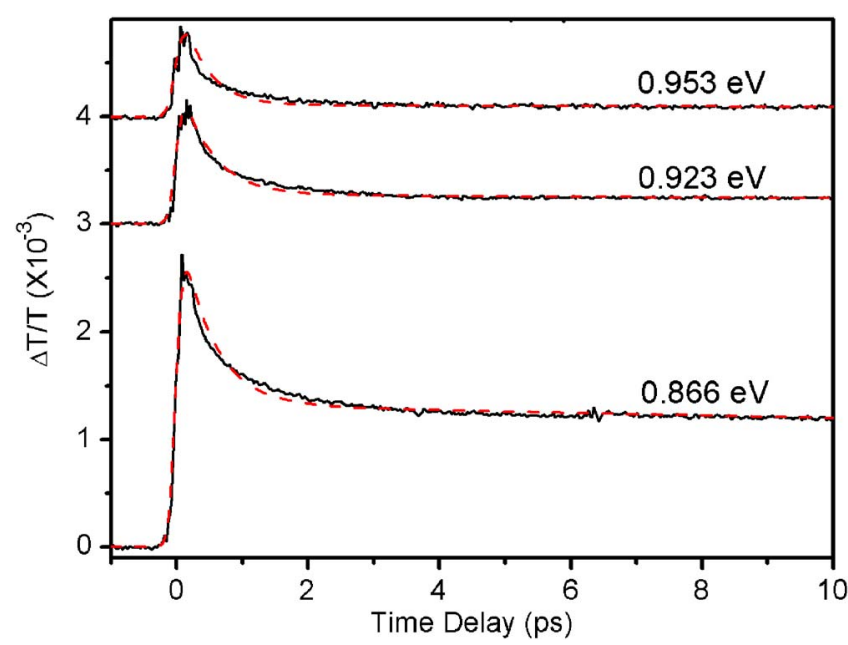

FIG. 1. (Color online) Measured transient transmission changes (solid lines) as functions of time delay at central wavelengths of $1300 \mathrm{~nm}(0.953 \mathrm{eV})$, $1340 \mathrm{~nm}(0.923 \mathrm{eV})$, and $1430 \mathrm{~nm}(0.866 \mathrm{eV})$ with a fixed photoexcited carrier density of $1.4 \times 10^{16} \mathrm{~cm}^{-3}$. All traces are in the perturbative regime. Traces are vertically displaced for clarity. Dotted lines are convolution fits.

the perturbative regime, thus attributing the fast decay component to the external carrier thermalization process (carrier cooling) and the slower decay component to the carrier recombination process. ${ }^{4,12}$ In these experiments, it is worth emphasizing that the femtosecond time-resolved pump-probe transmission technique, unlike some other time-resolved experimental schemes ${ }^{6,7}$ is a straightforward way with a high resolution to observe the ultrafast hot carrier relaxation and is capable of monitoring the time evolution of a carrier population at a specific probe photon energy. With excitation conditions carefully controlled in the perturbative regime, the complicated nonlinear mechanisms that participate in carrier relaxation can be avoided.

Compared to the previously reported carrier thermalization times at high carrier density, which were all found to be a few picoseconds slower-or even more- ${ }^{3,5,12}$ our observed carrier external thermalization time of $400( \pm 100)$ fs is much faster and approaches the theoretical electron-LO phonon scattering time. ${ }^{2}$ The study by Wen et al. ${ }^{5}$ reported $1.4( \pm 0.16)$ ps carrier cooling time taken in the perturbative regime at the high doping density of $6 \times 10^{18} \mathrm{~cm}^{-3}$ and concluded that screening of the electron-LO phonon interaction by dense plasma should reduce hot carrier cooling rate. With a reduced background doping density of $3.8 \times 10^{18} \mathrm{~cm}^{-3}$ in this study, one would thus expect a much faster carrier cooling rate. Nevertheless, because the screening effect is always confused with the hot phonon effect, it is highly desirable to discuss the role of the hot phonon effect in our current study. The hot phonon effect, resulted from nonequilibrium phonon populations causing phonon emission bottleneck, is related to the density and excess energy of the photoexcited carriers. Whereas, the screening effect, resulted from dense electronplasma, is related to the total carrier density, including background doping carriers and photoexcited carriers. For the data taken in the perturbative regime, the hot phonon effect is not expected to take place. Therefore, with the background doping density reduced to near the critical density of screening in $\mathrm{InN},{ }^{5}$ the dramatic decrease of carrier cooling time 


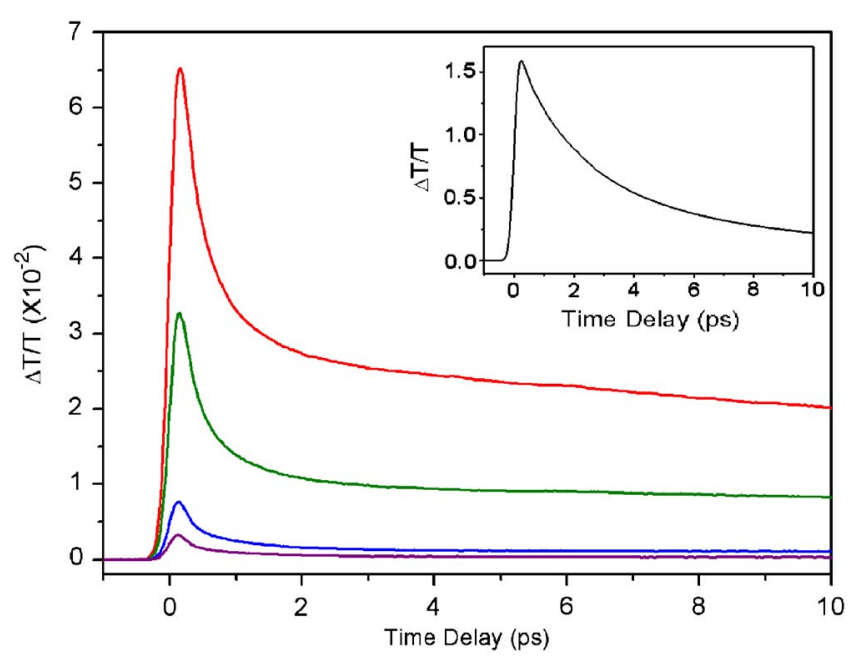

FIG. 2. (Color online) Measured transient transmission changes as functions of time delay at the excitation photon energy of $0.953 \mathrm{eV}$ with photoexcited carrier densities of (bottom to top) $4.7 \times 10^{16}, 9.6 \times 10^{16}, 3.7 \times 10^{17}$, and $7.2 \times 10^{17} \mathrm{~cm}^{-3}$. The inset shows the transient transmission change at the same photon energy with a high photoexcited carrier density of 4.2 $\times 10^{18} \mathrm{~cm}^{-3}$.

from $1.4( \pm 0.16)$ ps (Ref. 5) to $400( \pm 100)$ fs (taken in the perturbative regime) should thus be attributed to the reduction of the screening effect.

To quantitatively characterize the observed carrier cooling, we adopt a static-screening-approximation-based model, in which the screened Fröhlich interaction can be well described. ${ }^{13,14}$ In this model, the phonon emission rate is proportional to $\left[1+\left(N / N_{c}\right)^{2}\right]^{-1}$, where $N$ is carrier density and $N_{c}$ is critical carrier density, determined by the resonance condition of the plasmon with the LO phonon. ${ }^{15}$ For InN, $N_{c}$ can be calculated as $1.5 \times 10^{18} \mathrm{~cm}^{-3}$. Although our measured carrier cooling rate, a phenomenological parameter, is different from the phonon emission rate, the energy loss rate and the phonon emission rate is quite proportional. ${ }^{15}$ According to the static-screening approximation, when the carrier density decreases from $6 \times 10^{18}$ to $3.8 \times 10^{18} \mathrm{~cm}^{-3}$, the LO phonon emission rate should increase with a factor $\sim 2.3$. The threefold increase of the experimentally observed carrier cooling rate thus agreed reasonably well with the theoretical prediction, supporting the dominant role of the carrier screening reduction for the observation of the shortened carrier cooling time.

On the other hand, nonequilibrium phonon effects are always expected to play an important role in $\mathrm{InN}$ under high excitation, such as those in $\mathrm{GaN}$ or $\mathrm{ZnO}$ material systems. ${ }^{16,17}$ In this studied InN sample, since the screened carrier cooling time $400( \pm 100)$ fs is now shorter than the previously reported 0.7 ps RT LO phonon lifetime by a Raman measurement, ${ }^{18}$ the hot phonon effect was thus observed under high-intensity and high-energy photon excitations. As a result, we further investigated the hot carrier thermalization by changing the excitation intensity and wavelength to control the amount of phonon populations. Figure 2 shows the measured transient transmission traces, taken at a central wavelength of $1300 \mathrm{~nm}(0.953 \mathrm{eV})$, and that the excess energy of photoinjected electrons is at least three times the LO phonon energy, with photoexcited carrier densities of 4.7

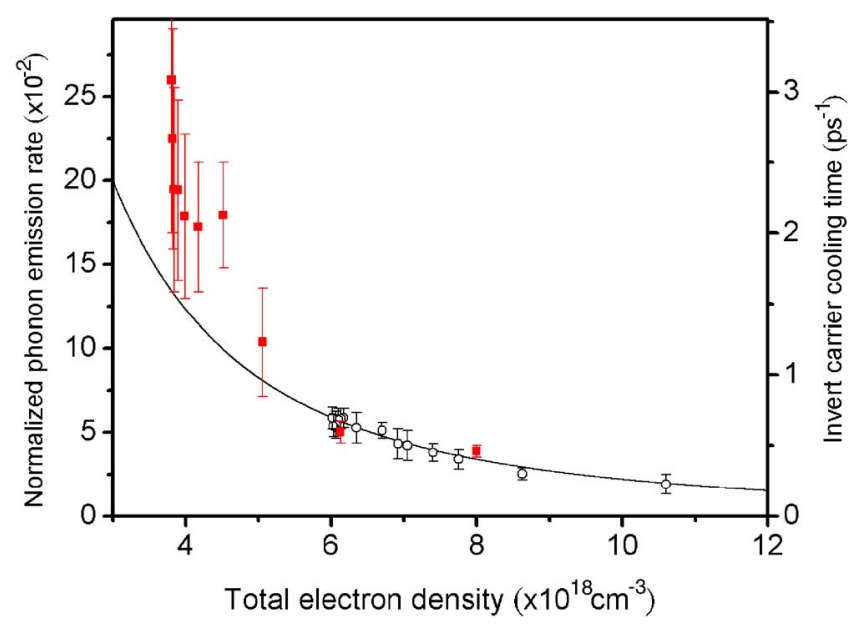

FIG. 3. (Color online) Normalized LO phonon emission rate (solid line) and the inverse of the carrier cooling time at a central wavelength of $1300 \mathrm{~nm}$ (open circles and full rectangles) as functions of total electron density. This figure was modified from Ref. 5, in which a detailed description can be found. The open circles represent the data taken in the previous study which had a higher background doping density of $\left(6 \times 10^{18} \mathrm{~cm}^{-3}\right)$. The solid squares represent the data taken in this study which had a lower background doping density $\left(3.8 \times 10^{18} \mathrm{~cm}^{-3}\right)$.

$\times 10^{16}, \quad 9.6 \times 10^{16}, \quad 3.7 \times 10^{17}, \quad 7.2 \times 10^{17}, \quad$ and $\quad 4.2$ $\times 10^{18} \mathrm{~cm}^{-3}$. When the optical excitation intensity was increased, no significant delay on the carrier cooling process was found unless the photoexcited carrier density reached close to the order of $10^{18} \mathrm{~cm}^{-3}$, comparable to the background doping density of $3.8 \times 10^{18} \mathrm{~cm}^{-3}$, when influence of the electron-plasma screening effect becomes apparent. No hot phonon effect was thus observed under our specific experimental condition and sample. One possible reason for this observation is that the LO phonon lifetime in this specific sample could be even shorter than the observed carrier cooling time, and consequently we are not capable of observing this even faster hot phonon effect. In fact, a recent study using the sample, fabricated by the same grower under a similar condition, on the phonon dephasing dynamics in $\mathrm{InN}$ indicated that the LO phonon dephasing time (or LO phonon lifetime) is shorter than $300 \mathrm{fs} .{ }^{8,9}$ With a relatively slower $400( \pm 100)$ fs screening-dominated carrier cooling time, the faster hot phonon effect thus does not play a significant role in the determination of the carrier cooling rate.

Except for the power dependent study under low photoexcitation, it is also desirable to verify if the high excitation data are found in a previous study ${ }^{5}$ based on a screening effect. Continuing using the same static-screening model described in the previous paragraph, in Fig. 3 we show the LO phonon emission rate and the measured inverse carrier cooling time as functions of the total electron density. In addition, the result of the previous report (open circles) was also shown for comparison. ${ }^{5}$ It should be noticed that the thickness of this sample is much greater than the optical skin depth, which implies the nonuniformity of the photoexcited carrier densities. Under high excitation, the observed dynamics, in fact, was contributed from regions with various electron densities and thus have mixed behaviors. Under these circumstances, we used an average carrier density to represent the observed mixed phenomena of carrier cooling. As 
can be seen in this figure, the high density data were in good agreement not only with the previous study but also with the trend of the screening model. The slight deviation in the low density data from the model can be attributed to the fact that the static-screening model is known to overestimate reduction in the LO phonon emission rate when the plasmon frequency $\omega_{p l}$ is close to the LO phonon frequency $\omega_{\mathrm{LO}}(1.5$ $\times 10^{18} \mathrm{~cm}^{-3}$ ) due to dynamic Coulomb interactions. ${ }^{15}$

\section{CONCLUSION}

We used the femtosecond pump-probe technique to study hot carrier relaxation dynamics in InN at RT. With a low background doping density, the observed external thermalization time (carrier cooling time) 400( \pm 100$)$ fs agreed with the expectation based on the Coulomb screening effect. Besides, the hot phonon effect, previously considered to be the cause of the reduction of the hot carrier relaxation rate, was not observed, which agreed well with a recent report that the LO phonon lifetime could be shorter than $300 \mathrm{fs}$ in specific InN samples.

\section{ACKNOWLEDGMENTS}

This work is sponsored by the National Science Council of Taiwan under NSC 96-2120-M-002-014. Y.-C.W. acknowledges the support of the MediaTek Fellowship.
${ }^{1}$ V. Yu. Davydov, A. A. Klochikhin, R. P. Seisyan, V. V. Emtsev, S. V. Ivanov, F. Bechstedt, J. Furthmuller, H. Harima, A. V. Mudryi, J. Aderhold, O. Semchinova, and J. Graul, Phys. Status Solidi B 229, R1 (2002). ${ }^{2}$ D. Zanato, N. Balkan, B. K. Ridley, G. Hill, and W. J. Schaff, Semicond. Sci. Technol. 19, 1024 (2004)

${ }^{3}$ F. Chen, A. N. Cartwright, H. Lu, and W. J. Schaff, Appl. Phys. Lett. 83, 4984 (2003)

${ }^{4}$ V. Pačebutas, G. Aleksejenko, A. Krotkus, J. W. Ager III, W. Walukiewicz, H. Lu, and W. J. Schaff, Appl. Phys. Lett. 88, 191109 (2006).

${ }^{5}$ Y.-C. Wen, C.-Y. Chen, C.-H. Shen, S. Gwo, and C.-K. Sun, Appl. Phys. Lett. 89, 232114 (2006).

${ }^{6}$ T.-R. Tsai, C.-F. Chang, and S. Gwo, Appl. Phys. Lett. 90, 252111 (2007).

${ }^{7}$ D.-J. Jang, G.-T. Lin, C.-L. Wu, C.-L. Hsiao, L.-W. Tu, and M.-E. Lee, Appl. Phys. Lett. 91, 092108 (2007).

${ }^{8}$ Y.-M. Chang, H. W. Chu, C.-H. Shen, and S. Gwo, Appl. Phys. Lett. 90, 072110 (2007).

${ }^{9}$ Y.-M. Chang and S. Gwo, J. Appl. Phys. 102, 083540 (2007).

${ }^{10}$ S. Gwo, C.-L. Wu, C.-H. Shen, W.-H. Chang, T. M. Hsu, J.-S. Wang, and J.-T. Hsu, Appl. Phys. Lett. 84, 3765 (2004).

${ }^{11}$ R. Tommasi, P. Langot, and F. Vallée, Appl. Phys. Lett. 66, 1361 (1995).

${ }^{12}$ R. Ascázubi, I. Wilke, S. Cho, H. Lu, and W. J. Schaff, Appl. Phys. Lett. 88, 112111 (2006).

${ }^{13}$ E.-J. Yoffa, Phys. Rev. B 23, 1909 (1981).

${ }^{14}$ M. A. Rodriguez, J. L. Carrillo, and J. Reyes, Phys. Rev. B 35, 6318 (1987).

${ }^{15}$ J. H. Collet, Phys. Rev. B 39, 7659 (1989).

${ }^{16}$ C.-K. Sun, F. Vallée, S. Keller, J. E. Bowers, and S. P. DenBaars, Appl. Phys. Lett. 70, 2004 (1997).

${ }^{17}$ C.-K. Sun, S.-Z. Sun, K.-H. Lin, K. Y.-J. Zhang, H.-L. Liu, S.-C. Liu, and J.-J. Wu, Appl. Phys. Lett. 87, 023106 (2005).

${ }^{18}$ J.-W. Pomeroy, M. Kuball, H. Lu, W.-J. Schaff, X. Wang, and A. Yoshikawa, Appl. Phys. Lett. 86, 223501 (2005). 